\title{
Erratum to: Bone turnover markers in patients with prostate carcinoma: influence of sex steroids levels
}

\author{
Mariela Varsavsky • Rebeca Reyes-García • \\ Antonia García-Martín · Pedro Rozas-Moreno • \\ Rocío González-Ramírez • Manuel Muñoz-Torres
}

Published online: 14 September 2013

(C) The Japanese Society for Bone and Mineral Research and Springer Japan 2013

Erratum to: J Bone Miner Metab

DOI 10.1007/s00774-013-0466-5

The correct name of the fifth author should be given as Rocío González-Ramírez, not González-Ramírez Rocío.

We apologize for the error.

The online version of the original article can be found under doi:10.1007/s00774-013-0466-5.

M. Varsavsky · R. Reyes-García ( $\varangle)$ · A. García-Martín ·

P. Rozas-Moreno $\cdot$ M. Muñoz-Torres

Bone Metabolic Unit, Endocrinology Division,

Hospital Universitario San Cecilio, Granada, Spain

e-mail: rebecarg@yahoo.com

M. Muñoz-Torres

e-mail:mmt@mamuto.es

M. Varsavsky

Endocrinology Division, Hospital Sant Pau i Santa Tecla,

Tarragona, Spain

P. Rozas-Moreno

Endocrinology Division, Hospital General Universitario de

Ciudad Real, Ciudad Real, Spain

R. González-Ramírez

Fundación para la Investigación Biosanitaria de Andalucía

Oriental-Alejandro Otero (FIBAO), Granada, Spain

M. Muñoz-Torres

Servicio de Endocrinología y Nutrición, Hospital Universitario

San, Cecilio. Av. Dr. Oloriz 16, 18012 Granada, Spain 